\title{
Inertial propagation of streamers after external voltage termination
}

\author{
Alexander V. Shelobolin \\ P.N. Lebedev Institute of Russian Academy of Science, Moscow, Russia
}

\section{Email address:}

shell@sci.lebedev.ru

\section{To cite this article:}

Alexander V. Shelobolin. Inertial Propagation of Streamers after External Voltage Termination. American Journal of Modern Physics. Vol. 2, No. 5, 2013, pp. 242-247. doi: 10.11648/j.ajmp.20130205.11

\begin{abstract}
The fundamental processes of streamer propagation in the streamer chamber after external voltage termination are considered on the basis of a plasma-waveguide model of gas electric breakdown. The model analyses the time dependence of streamer radiation under the mentioned conditions. The velocity and time scales of the process have been defined, and the electron density is estimated in the plasma waveguide formed before the external voltage termination. Earlier shortcomings of theories of this phenomenon are corrected.
\end{abstract}

Keywords: Gas Electric Breakdown, Voltage Termination, Streamer Pair, Nonlinear Plasma-Waveguide Model

\section{Introduction}

Among the phenomena of electric gas breakdown (EGB) in atmospheric-pressure gases the inertial propagation of streamers is the least studied one, and has not been adequate explained so far. The phenomenon has been studied in a streamer chamber (SC) [1], i.e. under the initiation of a streamer pair in the middle of an interelectrode gap by an external elementary particle. In the process of streamer stop streamer path of $\sim 1 \mathrm{~cm}$ and a flare have been observed

The mentioned scale of the inertial streamer path significantly increases the spatial scale of electron pulse relaxation, and in this connection arises the problem to explain the interelectrode medium anisotropy after external voltage termination. In addition, the registered flare has the maximum at the moment of streamer full stop. This leads to another problem, in relation to the necessity to explain the streamer propagation without using a hypothesis on the neutral gas photoionization by streamer self-radiation, and establishing the reason of such synchronization.

The prevailing theory of EGB considers a collisional ionization of the gas molecules by electrons in the vicinity of the wave head to be the main mechanism of wave propagation breakdown, and as an additional one - the gas ionization by the wave head optical radiation. So, even at the level of a qualitative analysis of the experiment [1] one encounters insuperable difficulties.
From the simplest qualitative considerations there appears that after external voltage termination in the chamber the interelectrode space becomes isotropic, and, correspondingly, the spatial scale of streamer electron pulse relaxation turns to be equal to the electron free path in an atmospheric gas, i.e. about several micrometers. This must correspond to the time scale of several picoseconds. The time scale of the streamer plasma energy relaxation should be greater than what could be inferred from the relation between the atom and electron mass. So, previous models assumed streamer stop after external voltage termination at a distance of several micrometers in several picoseconds and a monotonously decreasing de-excitation of the stored energy during $\sim 10 \mathrm{~ns}$.

As far as the mentioned estimates are concerned, the discussed experiment showed more than a three-fold difference in the free path and a qualitative coincidence in the de-excitation time. However, there is no complete coincidence between the estimates and experiment, since the estimate assumed a monotonously decrease in streamer brightness, and the experiment showed an increase followed by decrease. The experimental power-time function $I(t)$ has a standard form corresponding to the power released in the relaxing oscillating circuit plus constant background radiation.

To eliminate some of the contradictions the authors [1] have proposed a new model of streamer propagation, a model of longitudinal electromagnetic oscillation along the channel of streamer pair [2]. They assumed a no collisional 
approximation for the streamer plasma, and, according to the estimates given in [2], inferred a corresponding electromagnetic field oscillation frequency of $10^{11}-10^{12} \mathrm{~s}^{-1}$. In [3] the authors have expressed their doubt concerning the admissibility of such an approximation.

Such an objection can be accepted, since the frequency of the streamer pair neck in the SC observed in the experiment [4] performed by the same authors under practically the same conditions is essentially lower than the noted one. However, the main argument against the model [2] lies in the fact that the model does not explain the dynamics of streamer radiation after external voltage termination. Along with [2], the authors [1] returned to the consideration of the phenomenon in [5], and tried, on the basis of the energy balance, to give an explanation to the dependence of the streamer length growth $\Delta \mathrm{l}$ on the initial length $\mathrm{l}_{0}$ and initial field intensity $\mathrm{E}_{0}$, i.e. $\Delta \mathrm{l}=\mathrm{f}\left(\mathrm{E}_{0}, \mathrm{l}_{0}\right)$. Such an approach seems to be purely qualitative, as the terms of the energy balance in this problem can-not be defined with the needed degree of reliability and accuracy.

In [6] one can find additional arguments in favor of the model [2], however, the experimental quantitative data [1] has not been used. The most perfect model of the phenomenon considered in [7], which is based on a number of experimentally unconfirmed assumptions, gives an approximate ratio $\Delta \mathrm{l} \sim \mathrm{l}_{0} \cdot \mathrm{E}_{0}{ }^{1 / 3}$. The inaccuracy of this ratio comes from the fact that the experimentally found degree $1_{0}$ is smaller than unity. In all the papers concerned with the analysis of streamer propagation after external voltage termination $[2,5,7]$ the parameters of streamer radiation have not been taken into account, and, of course, the fact of the streamer stop at the moment of its maximum radiation has not been also taken into account. The unsolved problems are listed in most detail in [8], but the time dependence of power radiation obtained in [1] has not attracted the attention of the authors. The cause of anisotropy of the interelectrode space after external voltage termination has not attracted the attention of the authors of the mentioned models as well.

So, the streamer inertial path has been explained somehow $[2,5,7]$, but the streamer radiation time structure, the synchronization of the radiation with the process of inertial propagation and anisotropy of the interelectrode space after external voltage termination have not been explained so far. Moreover, one can state that this is impossible within the framework of the modern dominating concepts of the EGB wave propagation.

Paper [3] presents a review of the four existing streamer models and five leader ones. The difference criterion of the models is the difference in the hypothesis of the EGB wave propagation mechanism. Taking into account an additional leader model given in [3], and a subsequent paper [9], we have got at present seven leader models. Such a number of EGB models is due to the fact that there exists a lot of experiments which are not explained within the framework of the prevailing semi-empirical formulation of the problem.

The mentioned experiments will be named the fundamental ones. The fundamental experiments include not only the problem of a ball lightning, but the experiments in the conventional sections of EGB as well. The avalanche section covers the experiments on the observation of the ion transverse dip on the avalanche profile, the avalanche spatial periodicity in the multiavalanche processes, i.e. the avalanche equidistance [10, 11], and the avalanche velocity limitation from below by the value close to $10^{7} \mathrm{~cm} / \mathrm{s}$.

The semi-empirical models face insuperable difficulties in the interpretation of an avalanche-streamer transition, since in the corresponding experiments the momentum conservation law for mechanical particles is fulfilled. So, in [12] the streamer pair is formed and developed from the avalanche symmetrically in the reference system associated with the avalanche. In the streamer chamber [4] a similar pair is formed symmetrically in the laboratory reference system. In addition, at the avalanche-streamer transition the EGB wave diameter decreases two-three-fold, and this cannot be explained by the magnetic compression force [13, $14]$.

The considered effect of streamer inertial propagation is also a fundamental EGB experiment, as a semi-empirical formulation of a problem is unable to give an explanation to the above-mentioned effects. In the leader section of the EGB there are also the experiments that can-not be explained within the framework of conventional semiempirical models.

In order to overcome the contradictions the EGB nonlinear plasma-waveguide model (NPWM) is necessarily introduced. The model is based on three main statements:

- All the EGB waves are soliton.

- The EGB wave is the electric field longitudinal surface wave in the plasma waveguide.

- The equation describing the EGB wave propagation along the external electric field is the sine-Gordon equation for the electric potential.

The first point of this model is based on the noted symmetry of the avalanche-streamer transition and is a strict statement, as applied to the avalanches and streamers. An extrapolation of this statement to the leaders is as yet a hypothesis. The second statement of the NPWM is based on the second property of the avalanche-streamer transition. As seen from [15], the change in the EGB wave diameter is possible when the skin-layer thickness at the plasma waveguide surface turns to be smaller than the waveguide diameter, and in the vicinity of the waveguide axis there appears a dip at the electron density radial profile, and the surface wave goes from the external to the inner cylindrical surface of the profile.

The necessity of a waveguide approach is also due to the fact that the time parameter of the volume wave attenuation in plasma is of the order of inverse value of the electronneutral particle collision frequency [16]. This means that at the wave frequency smaller than the frequency of electronatom collisions, the wave is attenuated during the time shorter than the natural period, and one automatically loses 
the phase vibrational structure and the necessity to use this notion in the description of the EGB waves. Just that very case has urged the authors [2] to seek for the explanation of the discussed effect in collision less approximation, i.e. within the range of the waves with the frequency exceeding the frequency of electron-atom collisions. However, one needs essentially smaller frequencies to explain the synchronization of streamer and radiation space motion.

It should be noted that a soliton approach to the description of the EGB waves has been in use earlier [17, 18], however, the Korteweg-de Vries equation has been used there as the basic one, and the process of avalanchestreamer transition has not been qualitatively covered. Moreover, only the sine-Gordon equation has found the experimental justification [19], although just for the EGB experiment with laser initiation. Though, the Korteweg-de Vries equation is in better agreement with the experiment in case of the EGB leader mechanism. On the whole, from the three statements of NPWM just the first one is sufficiently well justified for the EGB avalanche and streamer waves. The second and the third statements still show signs of a hypothesis. In this connection the aim of the present paper is also to substantiate the noted statements, although the main task of this work is to resolve the contradictions between theory and experiment.

\section{Experimental Results and Interpretation Drawbacks}

The authors have insufficiently detailed [1] the conditions of the experiment. We'll try to restore the experimental conditions using the papers of the authors.

The construction of a streamer chamber (SC) used in [1] has been close to the standard ones producing a pulsed spatially homogeneous electric field in the gas interelectrode space (see, e.g., [11]). The start of streamers in the SC was initiated by the gas ionizing particle injected into the interelectrode space. If the particle enters along the field line, then a pair of streamers appears in the interelectrode space. If the particle comes perpendicular to the field line then there appears a series of streamer equidistant pairs. In [1] the authors used the second method, and this provided the appearance of several tens of streamer pairs. The authors [1] presented the arithmetic mean parameters of the streamers or distribution histograms, and the radiation has been integrally measured within the SC volume. The type of gas ionizing particle, its energy and the type of a radioactive source used in $[1,4,5]$ for the SC calibration are not indicated. The particle coordinate between the electrodes is not indicated as well. Below we'll assume it to be a relativistic electron, which crosses the interelectrode space just in the middle.

The voltage was directed to the electrodes with a certain delay after the particle passed through the interelectrode space. The delay is not indicated in [1], however, in [5] it is underlined that an increase of the delay up to hundreds of microseconds does not influence the process of streamer development. For further analysis of greater importance is the fact that the leading edge of a voltage pulse lasts 2-10 ns, comparable with the time scale of streamer process. This hampers correct mathematical formulation of streamer development in the acceleration mode regime, and in the description of streamer breaking mode regime may cause inaccuracy in time measurements. The pulse trailing edge duration makes up $0.5 \mathrm{~ns}$, and this allows one to consider the process of voltage termination to be instantaneous.

In [1] the average velocity at the stage of deceleration was reported to be $6 \cdot 10^{7} \mathrm{~cm} / \mathrm{s}$. This value is in agreement with other parameters of the experiment representing the average for the first 5 nanoseconds after voltage termination. Time reading in [1] starts with the beginning of the voltage pulse. In the models discussed below the time reading from the trailing edge of the pulse is more convenient. All the experiments $[1,4,5]$ use neon at 750 Torr pressure, and this is favorable for the problem, since, in comparison to other gases, the electron-atom elastic interaction cross-section in neon weakly depends on the energy, and is not subjected to the Ramseur effect.

The mentioned experimental peculiarities [1] are shown in Figure 1. Using relative time scales the figure presents: time dependence of the external field voltage $E_{0}(t)$; simplified streamer length vs. time histograms $N(t)$; time dependence of streamer head coordinate $1_{0}(\mathrm{t})$ and its increment after voltage termination $\Delta \mathrm{l}$; time dependence of the wave instantaneous velocity describing the streamer propagation $\mathrm{v}(\mathrm{t})$ (its peculiarities will be explained below), and the time structure of streamer radiation along the whole interelectrode space $I(t)$. Solid lines illustrate the parameters recorded experimentally in [1] or [4], and the dotted lines - the calculation and hypothetical parameters. The figure shows also the time characteristics to be used below in the description and calculation.

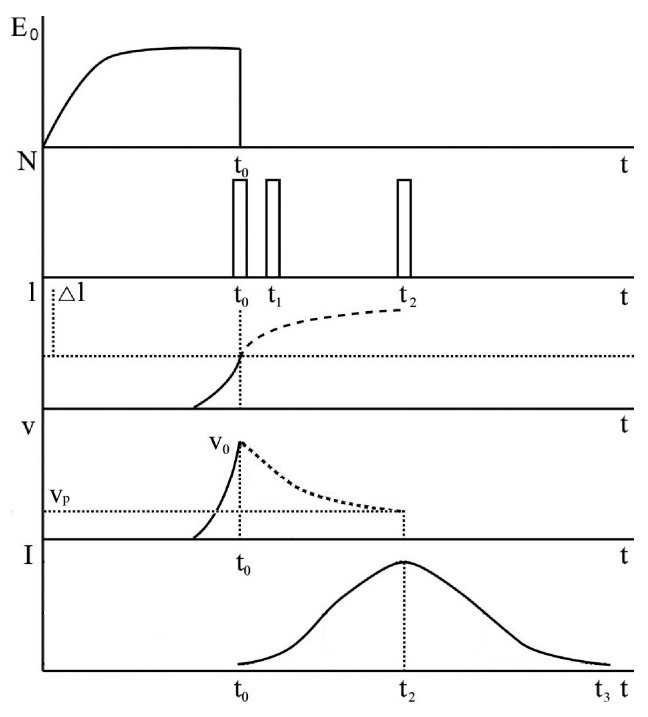

Fig 1. Dynamics of streamer acceleration and deceleration 
In detail the experimental results [1] are reduced to four items:

1. For a 30 ns voltage pulse, which provides in the SC the field amplitude intensity of $14.5 \mathrm{kV} / \mathrm{cm}$, the histograms plotted from the analysis of the photographs (the exposure time, $1 \mathrm{~ns}$ ) taken at the 30-th (time moment $\left.\mathrm{t}_{0}\right)$, 35-th $\left(\mathrm{t}_{1}\right)$ and 55-th $\left(\mathrm{t}_{2}\right)$ nanoseconds have shown at streamer average length of $5 ; 8$ and $10 \mathrm{~mm}$, respectively and this is equivalent to $3 \mathrm{~mm}$ increment of streamer at $5 \mathrm{~ns}$ after external voltage termination, and a $5 \mathrm{~mm}$ increment at 25 ns after external voltage termination.

2. The streamer extension after voltage termination grows if the streamer initial length at the moment of termination increases in accordance with the law slower than linearly. It is the author's opinion [1] that this dependence manifests itself more clearly than the dependence on external voltage.

3. After external voltage termination the streamers give a flare of 25 ns FWHM, and it weakly depends on the external field value. The duration of the leading edge of the flare is approximately $30 \%$ less than the trailing edge of the flare.

4. The streamer stops at maximum radiation, and its velocity drops from $10^{7} \mathrm{~cm} / \mathrm{s}$ to zero.

\section{General Picture of the Process within the Framework of NPWM}

A cylindrical ionized channel, a plasma waveguide, still exists in the interelectrode space just after voltage termination. According to the experiment, the lifetime of this channel is not less than $100 \mathrm{~ns}$. In the middle of the channel one can observe a region with enhanced ionization and excess charge accumulated at the stage of streamer development prior to voltage termination. After voltage termination it is discharged via the plasma waveguide and the whole SC similar to the oscillating circuit, and its power evolves according to the law: $\mathrm{I}(\mathrm{t})=\mathrm{I}_{0} \exp \left[-\left(\mathrm{t}-\mathrm{t}_{0}\right) / \tau_{1}\right]$ $\sin ^{2} \omega\left(t-t_{0}\right)$, where $\tau_{1}$ is the oscillating circuit relaxation time; $\omega$, the eigenfrequency.

Another relaxation process covers only the central part of the waveguide. The process includes:

1) The drop in temperature of the streamer pair plasma electrons Te by the law typical to neon, where the electronatom elastic interaction cross-section weakly depends on the energy [20]: $\mathrm{T}_{\mathrm{e}}=\mathrm{T}_{0}+\left(\mathrm{T}_{\mathrm{e} 0}-\mathrm{T}_{0}\right) \exp \left[-\chi v_{\mathrm{ea}}\left(\mathrm{t}-\mathrm{t}_{0}\right)\right]$, here $\mathrm{T}_{\mathrm{e} 0}$ represents the electron temperature at the moment of voltage termination; $\mathrm{T}_{0}$, the temperature of the ambient gas; $\chi=2 \mathrm{~m} / \mathrm{M}, \mathrm{m}$, the electron mass; $\mathrm{M}$, the mass of the atom; $v_{\text {ea }}$, the frequency of electron-atom collisions.

2) Development of a field running wave. The final phase velocity of the wave $v_{p}$ should be slightly greater than the thermal velocity of electrons at the maximum of distribution function. The velocity of streamer head $v$ after voltage termination should be exponentially approximated and should meet the boundary conditions of the time range, i.e.

$$
v(t)=\left(v_{0}-v_{p}\right) e^{-\frac{t-t_{0}}{t_{2}}}+v_{p}
$$

Here $\mathrm{v}_{\mathrm{p}}>\left(2 \mathrm{~T}_{0} / \mathrm{m}\right)^{0.5}=0.94 \cdot 10^{7} \mathrm{~cm} / \mathrm{s}$

At the moment of reaching the maximum $\mathrm{I}(\mathrm{t})$ the charge of the capacitive energy storage, i.e. the streamer pair, changes its sign, and this breaks the synchronism between the wave running through the waveguide and the medium. This, in turn, stops further streamer extension.

We can write three equations to describe the streamer inertial extension:

$$
\begin{gathered}
\Delta \mathrm{l}_{1}=\left(\mathrm{v}_{0}-\mathrm{v}_{\mathrm{p}}\right) \tau_{2}\left[1-\mathrm{e}^{-\frac{\mathrm{t}_{1}-\mathrm{t}_{0}}{\tau_{2}}}\right]+\mathrm{v}_{\mathrm{p}}\left(\mathrm{t}_{1}-\mathrm{t}_{0}\right)=0.3 \\
\Delta \mathrm{l}_{2}=\left(\mathrm{v}_{0}-\mathrm{v}_{\mathrm{p}}\right) \tau_{2}\left[1-\mathrm{e}^{-\frac{\mathrm{t}_{2}-\mathrm{t}_{0}}{\tau_{2}}}\right]+\mathrm{v}_{\mathrm{p}}\left(\mathrm{t}_{2}-\mathrm{t}_{0}\right)=0.5 \\
\mathrm{v}_{\mathrm{m}}=\left(\mathrm{v}_{0}-\mathrm{v}_{\mathrm{p}}\right) \frac{\tau_{2}}{\mathrm{t}_{1}}\left[1-\mathrm{e}^{-\frac{\mathrm{t}_{1}-\mathrm{t}_{0}}{\tau_{2}}}\right]+\mathrm{v}_{\mathrm{p}}=6 \cdot 10^{7}
\end{gathered}
$$

Equations (2) and (3) represent the result of subsequent integration (1) within the limits corresponding to the experimental conditions.

Equation (4) defines the streamer mean velocity during the $5 \mathrm{~ns}$ after voltage termination $\mathrm{v}_{\mathrm{m}}$. The three equations (2) - (4) form a system with relation to $\mathrm{v}_{0}, \mathrm{v}_{\mathrm{p}}$ and $\tau_{2}$. The solution for $\mathrm{v}_{\mathrm{p}}$ is limited by the range $0.94 \cdot 10^{7}<\mathrm{v}_{\mathrm{p}}<10^{7}$. The right boundary of the range is found from joint consideration of (2) and (3). After solving the system we get $\mathrm{v}_{0}=25.410^{7} \mathrm{~cm} / \mathrm{s}, \tau_{2}=1.034 \mathrm{~ns}, \mathrm{v}_{\mathrm{p}}=0.99 \cdot 10^{7} \mathrm{~cm} / \mathrm{s}$. .

To analyse the dependence of streamer extension on the initial length and the field value at the stage of streamer acceleration, i.e., $\Delta \mathrm{l}=\mathrm{f}\left(\mathrm{E}_{0}, \mathrm{l}_{0}\right)$, it is convenient to make use of Eq.(3).

The first term here depends (via $\mathrm{v}_{0}$ ) only on $\mathrm{E}_{0}$, and the second one depends only on $1_{0}$. The second term is analyzed quite easily by subsequently decoding the time parameters: i.e., $\mathrm{t}_{2}-\mathrm{t}_{0} \sim(\mathrm{LC})^{0.5} \sim \mathrm{C}^{0.5} \sim 1_{0}^{0.5}$, where $\mathrm{L}$ is the discharge circuit inductance; $\mathrm{C}$, the discharge circuit capacitance, which is equal to the capacitance of all streamer pairs and is proportional to their average length.

So, the ratio $\Delta \mathrm{l}=\mathrm{f}\left(\mathrm{l}_{0}, \mathrm{E}_{0}=\right.$ const $)$ must have a parabola as a summand, and this is close to the experiment [1]. A transition to other conditions in the experiment may lead to a change in the relation between the first and second terms in (3). In particular, an increase in L results in an increase of the interval $\left(t_{2}-t_{0}\right)$, and this will influence the relation between the terms in (3), which will concern not only the physics of the process, but the constructional characteristics of streamer chamber. 
The analysis of the first term in (3) turns to be more complicated, since $\mathrm{v}_{0}$ depends not only on $\mathrm{E}_{0}$ directly, but on the time delay of streamer formation, the shape and duration of the voltage pulse up to the pulse termination as well (see Fig.1). If the first interval may be related to $E_{0}$ and the shape of the leading edge of the pulse, the second interval presents the parameter determined by the experimenter beyond the frames of the discussed problem. This means that the mere formulation of a problem of comparison between the relative influence of the field and initial streamer length on the streamer stretching is not a universal one, and, thus, is not quite correct.

\section{Estimation of the Waveguide Ionization Density}

In [22] such estimation has been made from below, i.e., assuming that the waveguide radius is not less than three Debye lengths. We have an opportunity to carry out an estimation from above, i.e., based on the analysis of $\mathrm{I}(\mathrm{t})$.

For the convenience of error estimation the analysis will be made step by step:

1) From the experimental ratio $\mathrm{I}(\mathrm{t})$ one can get two parameters: the dimensionless shape parameter $\alpha=$ $4 \mathrm{~L} /\left(\mathrm{R}^{2} \mathrm{C}\right)$ and the time scale of the oscillating circuit $\mathrm{RC} . \mathrm{R}$ is the circuit resistance.

The parameter $\alpha$ illustrates the circuit operation regime. If $\alpha>1$, then we'll have the regime of damped sinusoid. One can show

$$
\alpha=\sec ^{2} \pi \frac{t_{2}-t_{0}}{t_{3}-t_{0}}
$$

For $\mathrm{t}_{2}-\mathrm{t}_{0}=25 \mathrm{~ns}$, and $\mathrm{t}_{3}-\mathrm{t}_{0}=59$ ns we get $\alpha=17.8>1$, and this has been expected from a qualitative analysis of $\mathrm{I}(\mathrm{t})$

2) In the same way, from the analysis of the circuit operation in the mentioned regime one can get:

$$
R C=\frac{t_{3}-t_{0}}{\pi} \sin 2 \pi \frac{t_{2}-t_{0}}{t_{3}-t_{0}}
$$

For the mentioned time values we have $\mathrm{RC}=8.66 \mathrm{~ns}$. One should note: $\mathrm{RC}=\mathrm{r} / \mathrm{N} \cdot \mathrm{cN}=\mathrm{rc}$, where $\mathrm{N}$ is the number of streamers; $r$, the resistance of one of the waveguide channels; $c$, the capacitance of one streamer pair. Making use of [22], one can estimate the capacity of one streamer pair $\mathrm{c}=0.28\left(\mathrm{l}_{0}+\Delta \mathrm{l}\right) / \ln (2 \mathrm{l} / \mathrm{D}) \mathrm{pF}=0.04 \mathrm{pF}$. Then, $\mathrm{r}=\mathrm{RC} / \mathrm{c}=2.1 \cdot 10^{5} \mathrm{Ohm}$.

3) As the waveguide conductivity is $\sigma=4 \mathrm{~d} /\left(\pi \mathrm{D}^{2} \mathrm{r}\right)=$ $\mathrm{e} \mu \mathrm{n}$, where $\mathrm{d}$ is the interelectrode space; $\mathrm{D}$, the waveguide diameter; e, the elementary charge; and $\mu$, the electron mobility in the given gas, then one can write for electron density $n$ :

$$
n=\frac{4 d}{\pi e \mu D^{2} r}
$$

At $\mathrm{d}=3.8 \mathrm{~cm} \mathrm{[4],} \mathrm{D}=10^{-2} \mathrm{~cm}, \mu=2 \cdot 10^{3} \mathrm{~cm}^{2} /(\mathrm{V} \cdot \mathrm{s})$ [5] we have $n=7 \cdot 10^{14} \mathrm{~cm}^{-3}$. The inaccuracy of the value comes from the inaccuracy of (RC), D, $\mu$ and, as a whole, does not exceed one order of magnitude, which is quite acceptable for the parameter $n$. Such value of electron density satisfactorily coincides with the experimental data obtained in [14] sufficiently far from the streamer head.

\section{Conclusions}

1) The performed investigation has revealed that the experiment [1] confirms the hypothesis on the existence of a plasma waveguide connecting the electrodes after the external voltage termination. This conclusion is based on three statements. First, this is the only way to explain the anisotropy of interelectrode space after external voltage termination. Second, the discharge via the SC oscillatory circuit starts immediately after the voltage termination and lasts $100 \mathrm{~ns}$. Third, further streamer propagation in the SC presents a wave which runs through the waveguide and follows the synchronization of its phase velocity and the velocity of thermal motion of electrons of the medium. The change of the sign of a streamer pair explains an instantaneous stop of a streamer at the radiation maximum.

2) The diagnostics of electron density in the plasma waveguide, based on the analysis of the $I(t)$ dependence is in satisfactory agreement with direct density measurements in other independent experiments, and this confirms once more the validity of NPWM.

3) The NPWM satisfactorily describes the radiation process under inertial propagation of streamer as the radiation coming from the waveguide volume not occupied by streamer pairs, and allows further experiments to define more exactly the model ideas.

4) The model assumes insignificant influence of streamer eigenradiation on its dynamic characteristics.

5) The model does not allow one to give preference to the streamer initial length or the initial voltage in the experiment when estimating the inertial extension.

\section{References}

[1] Rudenko N.S and V.I. Smetanin. Streamer propagation after the voltage termination in chamber. Sov. Phys. Techn. Phys. 19, p.1616. 1975.

[2] N.S. Rudenko and V.I. Smetanin. Mechanism of streamer propagation based on plasma oscillations. Izvestiya vuzov. Fizika. v.7. p.34. 1977.

[3] A.F. D'yakov, Y.K. Bobrov, A.V. Sorokin and Y.V. Yrgelenas. Physical Concept of Electrical Breakdown of Gases (Moscow: MPEI) 1999.

[4] N.S. Rudenko and V.I. Smetanin. Investigation of large-gap 
streamer neon breakdown. Sov. Phys. - JETP. 34, 76. 1972.

[5] A.A. Vorob'ev, N.S. Rudenko, V.I.Smetanin. Spark chamber technique. Moscow, Atomizdat. 1978.

[6] L.P. Babich Fiz. Plazmy 7, 1419 [Sov. J. Plasma Phys. 7, 783] 1981.

[7] E.D. Lozansky and O.B. Firsov. Streamer propagation in a zero leading field. J. Eksp. Teor. Phys., v.2, 8, p.352. (JETP Lett.) 1976.

[8] A.N. Lagar'kov and I.M. Rutkevich. Ionization waves in electrical breakdown in gases. Springer-Verlag, Inc. 1994.

[9] A.V. Gurevich and K.P. Zybin. Runaway breakdown and electric discharges in thunderstorms. Phys. Uspekhi 44(11), 1119 - 1140. 2001.

[10] K.R. Allen, K. Phillips K. Cloud chamber study of electron avalanche growth. Proc. Roy. Soc., 274 A, 163. 1963.

[11] H. Raether. Electron Avalanche and Breakdown in Gases. Butterworths. London. 1964.

[12] K.H. Wagner. Electron avalanche-to-streamer transition investigated by means of image intensifier streak shutter technique. Proceedings of the Seventh International Conference «Phenomena in Ionized Gases». Beograd. 1965, V.1, p.571.

[13] H. Tholl H. Zs. Naturforsch. V.180, P.516. 1964.
[14] P. Stritzke, I. Sander and H. Raether Spatial and temporal spectroscopy of streamer discharge in nitrogen. J. Phys. D: Appl. Phys., Vol.10, p.2285. 1977.

[15] A.V. Shelobolin Quality factor of plasma waveguide and contraction of laser-induced breakdown in gases. Plasma Phys. Rep., 32, p. 880. 2006.

[16] A.N. Kondratenko. Plasma Waveguides (Moscow; Atomizdat). 1976.

[17] H. Jrenka and E. Barreto. Study of electron waves in electrical discharge channels. J. Appl. Phys. Vol.53, №5, p.3481. 1982.

[18] A.N. Lagar'kov and I.M. Rutkevich. Fiz. Plazmy 7, 1132. 1981. [Sov. J. Plasma Phys., 7622 (1981)]

[19] A.V. Shelobolin A.V. Diagnostics of Solitary Waves of Electric Breakdown in Gases. Journal of Russian Laser Research. V25, N5. p.440. 2004.

[20] V.E. Golant, A.P. Zhilinsky, I.E. Sakharov I.E. The principles of plasma physics. Atomizdat. Moscow. 1977.

[21] A.V. Shelobolin. Plasma-waveguide model of electric breakdown in gases Plasma Phys. Rep.., 29, 166. 2003.

[22] T.M. Bazelyan and Y.P. Raizer. Spark Discharge. Chem. Rubber Co. Press. New York. 1998. 